\title{
Submandibular gland chronic sialadenitis: A case report
}

\author{
Rubia Siddiqui ${ }^{1, *}$, G.S.Hashmi ${ }^{2}$, S.M. Rizvi ${ }^{3}$ \\ ${ }^{1}$ Ex. Assistant Professor, ${ }^{2,3}$ Associate Professor, Dept. of Oral and Maxillofacial Surgery, Dr. Ziauddin Ahmad Dental, College, \\ Aligarh Muslim University, Aligarh, Uttar Pradesh, India \\ *Corresponding Author: Rubia Siddiqui \\ Email: rubiasiddiqui@gmail.com
}

\begin{abstract}
Among various types of salivary gland diseases, sialadenitis is an infectious or inflammatory disorder. The course of sialadenitis may be acute or chronic in nature. The chronic conditions generally involve the parotid and submandibular glands. This report describes a case of chronic sialadenitis of submandibular gland in a 55-year-old female which was resistant with medicinal therapy and successfully managed surgically with no postoperative complications.
\end{abstract}

Keywords: Submandibular gland, Recurrent, Chronic sialadenitis, Excision, Histopathology.

\section{Introduction}

An inflammatory condition of salivary glands is known as sialadenitis. It may be acute, chronic or recurrent. The chronic conditions generally involve the parotid and submandibular glands. Children and adults are commonly affected. ${ }^{1}$ Among the major salivary glands, about $10 \%$ cases of sialadenitis is seen in submandibular gland. Old age, debilitated, and dehydrated patients are more prone to this disease. ${ }^{2}$ Sialadenitis may be caused by bacterial or viral infection, radiation and allergic reactions, trauma, and autoimmune disorders. ${ }^{3,4}$ Chronic conditions leads to swelling of the gland, pain, tenderness, trismus and occasionally fever. Antistaphylococcal antibiotic along with adequate rehydration should be advised. When tenderness subsided repeated massaging of the gland can be done. Here we report a case of a submandibular gland chronic sialadenitis in a 55-year-old female with its diagnosis and treatment.

\section{Case Report}

In the department of Oral and Maxillofacial Surgery, Dr. Z.A. Dental College and Hospital a 55year old female patient reported with the chief complaint of pain and swelling in the lower right side of the face for two months. Medical history was not relevant. The patient didnot have difficulty in eating and speaking. According to the patient, initially swelling was small in size and slowly increased to the present size of $4-3 \mathrm{~cm}$. The nature of pain was moderate to severe and it was radiating up to the level of head and neck. The patient consulted a private practitioner also but could not get relief. Diagnosis of chronic sialadenitis was based on careful consideration of patient history and thorough clinical and radiographic examination.

On clinical examination, a single ovoid swelling with diffuse margins was present in the right submandibular region. The anteroposterior extension of the swelling was $1.5 \mathrm{~cm}$ posterior to the chin upto the angle of mandible. Superoinferiorly it was extending
$1 \mathrm{~cm}$ below from the lower border of the mandible in the right submandibular region upto a distance of $3 \mathrm{~cm}$ inferiorly. The swelling was soft with a smooth surface and normal overlying skin color and temperature. It was nonreducible, noncompressible, nonpulsatile and not fixed to the overlying skin but tender on palpation. Discharge or sinus formation was absent. There was no difficulty in deglutition and swelling did not increase in size during meals. It was bimanually palpable. The neck was supple with no lymphadenopathy, no bruit or venous engorgement. Other intraoral findings were carious lower right first molar with considerable supragingival and subgingival calculus and stains. USG of the neck is suggesting that right submandibular gland is bulky and having hypoechoic echotexture with increased vascularity. ${ }^{5}$

CECT Neck showed a bulky submandibular gland of right side suggesting of sialadenitis of the gland (Fig. $1 \mathrm{a}, \mathrm{b})$. FNAC of the swelling reports the presence of inflammatory cells. Based on the above mentioned clinical and radiographic findings and FNAC report a diagnosis of chronic sialadenitis of submandibular was made. The patient was put on Tab AmoxycillinClavulanic acid $625 \mathrm{mg}$ tds, Tab Metrogyl 400mg tds, tab paracetamol $500 \mathrm{mg}$ bd and chlorhexidine mouthwash for 5 days. After 5 days of medication, the patient came with no significant improvement in pain and swelling. Also patient gave a history of the prior antibiotic cover so it was a case of chronic sialadenitis which was refractory to medicinal therapy so it was planned for excisional biopsy.

The patient was operated under general anesthesia. In right side, skin incision was given about $2 \mathrm{~cm}$ below from the lower border of the mandible. Skin and subplatysmal flaps were raised. The marginal mandibular nerve was identified and preserved. Facial vein and duct of the gland were identified and ligated. The submandibular gland along with the duct was removed completely. (Fig. 2) Microscopic examination of the excised sample showed dense chronic inflammation comprising mainly of lymphocytes with 
few dilated ducts with inflammation around it (Fig. 3a) suggestive of submandibular gland chronic sialadenitis. The patient was put on antibiotics and anti- inflammatory drugs and recalled after 1 week with follow up of 6 months. Healing was satisfactory without any postoperative complications. (Fig. 3b)

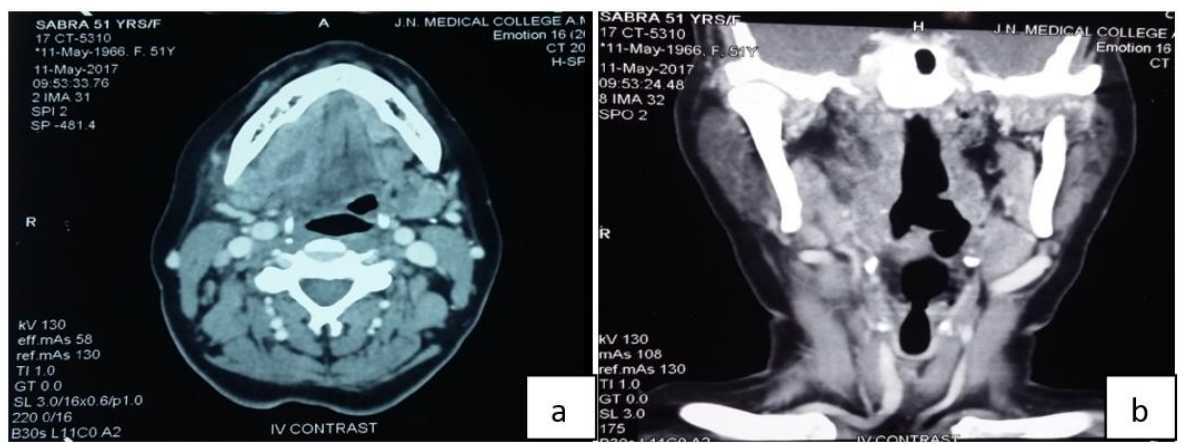

Fig. 1: (a) Axial and; (b) Coronal section of C.T. scan of the neck showing expansion of the right submandibular gland. Histopathological examination after surgical excision confirmed a chronic sialadenitis
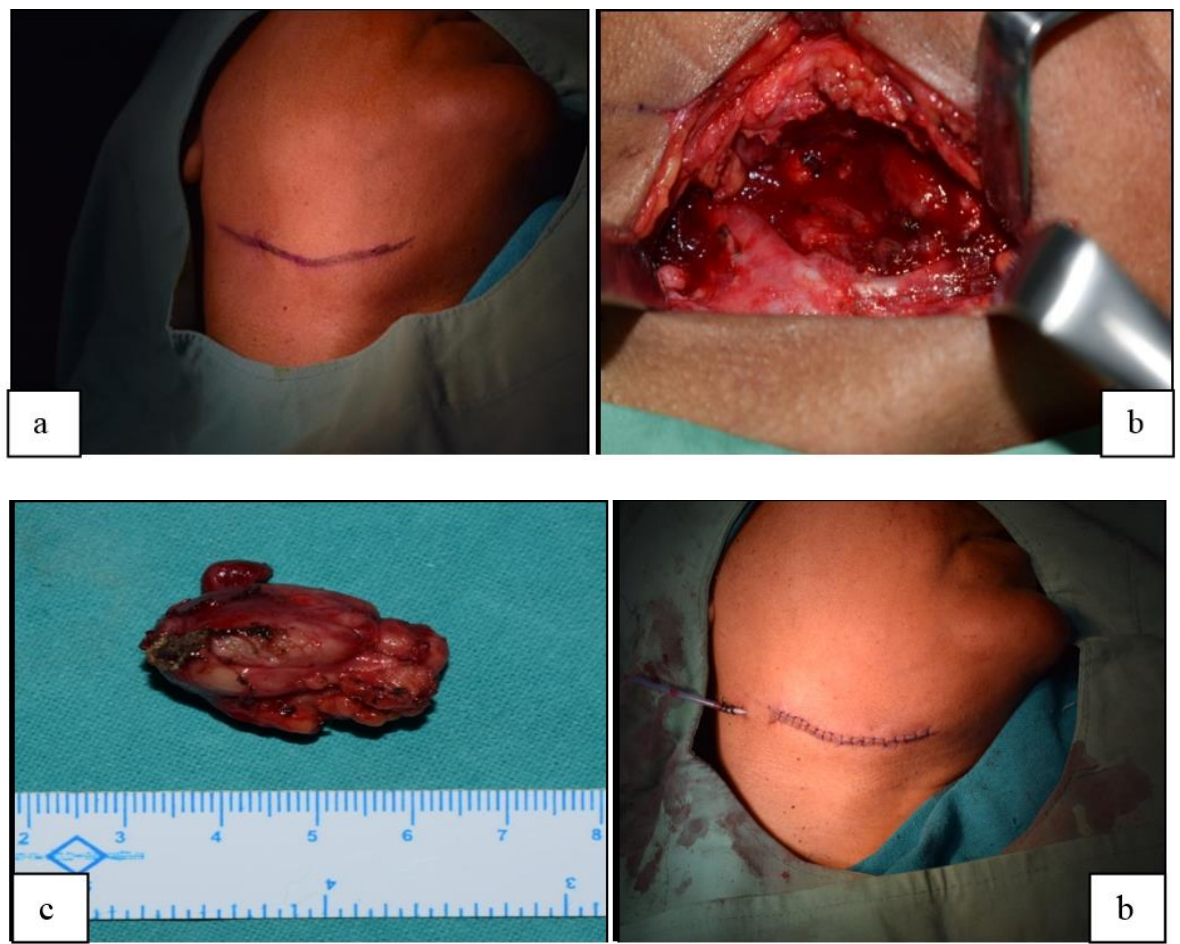

Fig. 2: (a) Lateral view of the right side of neck showing swelling i.r.t. right submandibular gland along with the incision mark; (b) Surgical site after excision of the submandibular gland; (c) Specimen of the submandibular gland; (d) Closed surgical site
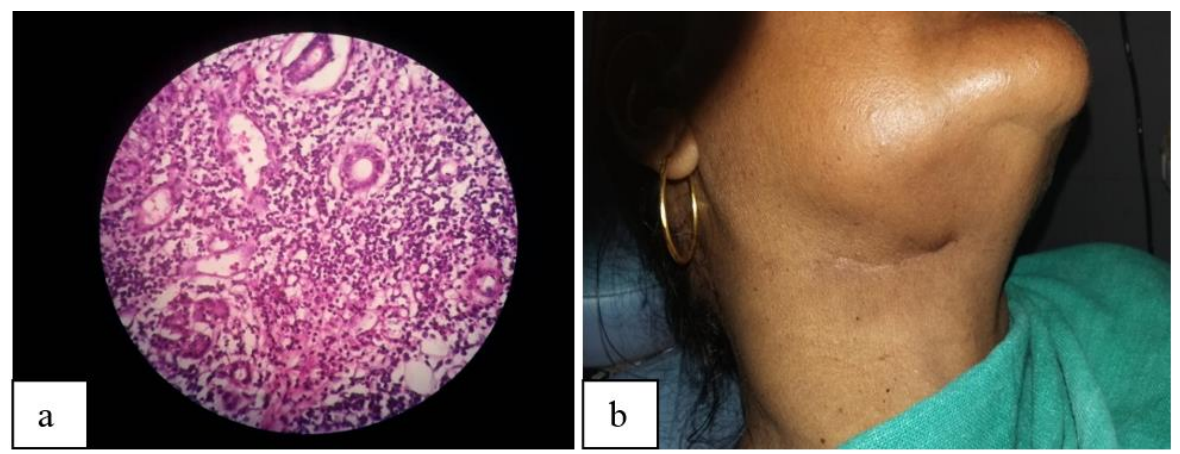

Fig. 3:(a) Photomicrograph of the submandibular gland sialadenitis showing dense lymphocytic infiltrate, (b) Follow up after three months. 


\section{Discussion}

In chronic sialadenitis pain and inflammation occurs in repeated episodes. It may be due to decreased salivary flow and salivary stasis. It most commonly affects the parotid gland. ${ }^{6}$ The initiating factors may include salivary duct obstruction from stones, scarring, strictures, compression by a tumor or foreign bodies. ${ }^{7}$

Patients with chronic sialadenitis are evaluated with the help of a proper history, physical and radiographic examination, culture, laboratory investigation, FNAC if indicated. ${ }^{7,8}$ Common symptoms are painless swelling, moderate to severe pain with glandular swelling accompanied by trismus and usually associated with swelling while eating. ${ }^{8}$ Patient discussed here was having most of the typical symptoms of chronic sialadenitis.

Physical examination shows enlargement of the gland. The swelling may subside in later stages of the disease. Bimanual intraoral and extraoral palpation distinguishes between the masses of the submandibular gland and the nonsubmandibular gland origin. ${ }^{9}$ The examination should focus on the predisposing factors like sialolith or stricture formation in order to remove the etiological factors.

The traditional radiographic diagnostic tools include plain radiographs (occlusal radiograph), sialography, ultrasound, and scintigraphy. Sialo-CT and magnetic resonance sialography are recently introduced diagnostic methods. ${ }^{10}$

Management of submandibular gland sialadenitis can involves a range of approaches, from conservative management to more aggressive surgical procedure. Generally if no cause is found treatment is conservative. It consists of anti-inflammatory medications, sialagogues, massage and hydration. Excision of salivary gland can be considered in extremely severe cases. This procedure is safe with a low rate of complication like xerostomia. ${ }^{11}$ In the present case, submandibular gland was excised with no postoperative complications in a follow up of 2 years.

\section{Conclusion}

Management of submandibular gland sialadenitis involves a wide range of approaches, from conservative to more aggressive surgical intervention. If sialadenitis is refractory to medicinal therapy then surgical intervention should be done.

\section{References}

1. P. J. Bradley, "Pathology and treatment of salivary gland conditions," Surgery. 2006;24(9):304-311.

2. G. Isacsson, A. Isberg, M. Haverling, and P. G. Lundquist, "Salivary calculi and chronic sialoadenitis of the submandibular gland: a radiographic and histologic study," Oral Surgery Oral Medicine and Oral Pathology. 1984;58(5):622-627.

3. Gayner SM, Kane WJ, McCaffrey. Infections of the salivary glands. In: Cummings CW, et al, eds.
Otolaryngology-Head and Neck Surgery. 3rd ed. St. Louis, Mo: Mosby; 1998;1234-46.

4. Rosai J. Major and minor salivary glands. In: Rosai J, ed. Ackerman's Surgical Pathology. Vol 1. St. Louis, 1996:815-56.

5. Alyas F, Lewis K, Williams M. Diseases of the submandibular gland as demonstrated using highresolution ultrasound. Br J Radiol. 2005;78(928):362-9.

6. Saunders JR Jr, Hirata RM, Jaques DA. Salivary glands. Surg Clin North Am. 1986;66(1):59-81.

7. Zou ZJ, Wang SL, Zhu JR, Wu QG, Yu SF. Chronic obstructive parotitis. Report of ninety-two cases. Oral Surg Oral Med Oral Pathol. 1992;73(4):434-440.

8. Rogers J, McCaffrey TV. Inflammatory disorders of the salivary glands. In: Flint PW, Haughey BH, Lund VJ. eds. Cummings Otolaryngology Head and Neck Surgery. 5th ed. Philadelphia, Pa.: Mosby Elsevier; 2010:11511161.

9. Hedge S, Praveen BN, Shishir RS, Ajila V. Sialadenitis of left submandibular salivary gland associated with giant sialolith - A case report. Indian J Med Res Pharm Sci. 2014;1:43-7.

10. Kim JP, Park JJ, Son HY, Woo SH. An unusual case of bilateral submandibular sialolithiasis. J Med Cases. 2012;3:106-9.

11. O'Brien CJ, Murrant NJ. Surgical management of chronic parotitis. Head Neck. 1993;15(5):445-449. 